\title{
A Method of Multi-Dimensional Information Spatial Partitioning and Intelligent Communication Path Planning of Metallurgical Equipment
}

\author{
Jianyi Kong ${ }^{1}$, Junwei Liu ${ }^{1}$, Yongbing Li $^{2}$, Xingdong Wang ${ }^{1}$, and Min Zhou ${ }^{1}$ \\ ${ }^{1}$ College of mechanical automation, Wuhan University of Science and Technology, Wuhan 430081 P. R. China \\ ${ }^{2}$ Wuhan Iron and steel enterprise, Wuhan 430080 P. R.China
}

Received: 1 Oct. 2012, Revised: 23 Dec. 2012, Accepted: 26 Dec. 2012

Published online: 1 Jun. 2013

\begin{abstract}
The process of metallurgical equipment information space is complex and diverse, including multi-level spatial information or spatial process factor. How to divide the metallurgical equipment information space, to plan its communication path intelligently and to enhance the level of intelligent equipment has become one of the metallurgical equipment systems' problems needing to be resolved. Aiming at enhancing the intelligence of iron and steel metallurgy equipment systems, the problems of the metallurgical equipment information space dividing and information communication path intelligent planning are taken as the research object. The metallurgical equipment information spatial characteristics (such as the space distribution, spatial morphology, spatial behavior, spatial scale effects and space dependence, etc) are compositely analyzed. Based on the cellular automata theory, ubiquitous information spaces of metallurgical equipment business process control and intelligent control are divided. Information communication path intelligent planning method of metallurgical equipment based on ant colony algorithm is explored. The practicality and effectiveness of the divided information spaces and designed algorithms are verified by simulation, which provides theoretical support to enhance the level of metallurgical equipment.
\end{abstract}

Keywords: Metallurgical equipments, cellular automata, composite analysis and partitioning of the information space, ant colony algorithm, intelligent planning of the communication path

\section{Introduction}

Metallurgical equipment intelligent is an intelligent manufacturing process under the ubiquitous-aware networking [1]. With the development of ubiquitous information environment in metallurgical industry, driving type of metallurgical equipment systems is changing from energy-driven to information-driven. Intelligent information processing technology is one of the foundation and important guarantee for the intelligent production of iron and steel metallurgy under information-driven production environment.

Intelligent information processing technology includes information collection technology, information data structures processing technology, information transmission technology and decision-making technology based on information. In the ubiquitous information environment, the critical and difficult points of the information transmission technology is network information space technology, which includes the construction of information network, the composite analysis of the information space, and information efficient transfer methods based on information space. H.B Max has advanced the information space theory that the information space is composed of information coding, abstraction and diffusion [2]. Every spot in knowledge resource space model is only to determine one or a class of knowledge resources by Zhen Lu [3]. The idea of time and space division is used to data-mining operation such as data classification, clustering and discovery of association rules, etc. by Zhengwu Yuan [4]. Forecasting model of electric load space distribution based on CA theory is proposed by Lixi Yang. The problems of electric load forecasting such as large amount of data, many uncertainties and related factors, three basic characteristics as time, space and attribute covering

\footnotetext{
* Corresponding author e-mail: 15697188659@wo.com.cn
} 
geographical and so on are solved by the model[5]. CA (Cellular Automata) theory is a general term for a class of mathematical models and methodological frameworkwhich is a discrete, infinite dimensional dynamical systems. It is an emerging branch of artificial intelligence [6]. A two-dimension cellular automata is used to develop the evolution rules of the wireless sensor network system topology in network technology. It ensures the coverage rate and connectivity of the network topology control, and extends the lifetime of the network[7]. The multi-level grid cellular automaton model of spatial information is proposed by Shuangfeng Wei[8]. The results of image segmentation provide the basis for target identification and tracking [9]. The CA is used to simulate the space boundary condition by Wang Min[10]. Multidimensional complexity is often ignored in spatial analysis[11]. The process of metallurgical equipment information space is complex and diverse, including multi-level spatial information or spatial process factor. The object of the metallurgical equipment information spatial analysis is the spatial relationships of the metallurgical equipment information. According to production data, process data, time attribute data, thematic attribute data and a variety of other information sources within metallurgy equipment information space, a spatial analysis model is established. Different forms of analysis are taken from the spatial dimensions, spatial location, spatial distribution and spatial form to extract and transfer the spatial information[12]. The analysis will gradually provide support for Information spatial decision-making. The ACO (Ant Colony Optimization) algorithm[13] has a clear advantage in realizing the fast approaching optimization of the ubiquitous cellular information space. It provides an avenue for intelligent matching of metallurgical equipment information. The ACO algorithm is often introduced into the three-dimensional space path planning problem of the robot in complex environments [14][15]. The space between the robot location (origin) and the destination point is subdivided into the three-dimensional grid, and the optimal path from the origin to the destination is found out[16]. The ACO algorithm is used in the self-organization services recommended network to improve success rate and recall rate of the service discovery [17]. The ACO algorithm is often introduced to solve the continuous space function optimization and the diffusion process route quickly optimization[18].

Aiming at enhancing the intelligence of iron and steel metallurgy equipment systemsthe information spatial transfer technology (one of the key factors affecting the overall intelligence of iron and steel metallurgy equipment systems) is studied. Based on the analysis of information sources and information flow of the metallurgical equipment, metallurgical equipment information space is built. The metallurgical equipment information space division method is explored based on the CA. The information communication path (within the metallurgical equipment information space) intelligent planning method based on the ACO algorithm is proposed.

\section{Composite Analysis of the Metallurgical Equipment Information Space}

The attribute data is generally used as a basis of division in traditional metallurgical equipment information space analysis, and the spatial relationships between the various data unit is ill-considered. Composite analysis of the metallurgical equipment information space not only considers the attribute information of the space units, but also increases the interdependence of the information space units. This makes the multi-scale information space unit changing from small to large appear in the information space. The information space units with highly spatial interdependence mix together to become a metallurgical equipment information space whit different spatial scales[19]. The spatial scale effect and spatial dependence are compositely analyzed based on the spatial distribution, spatial behavior, etc. Thus the space division and space optimization are carried out, and the spatial decision is supported finally.

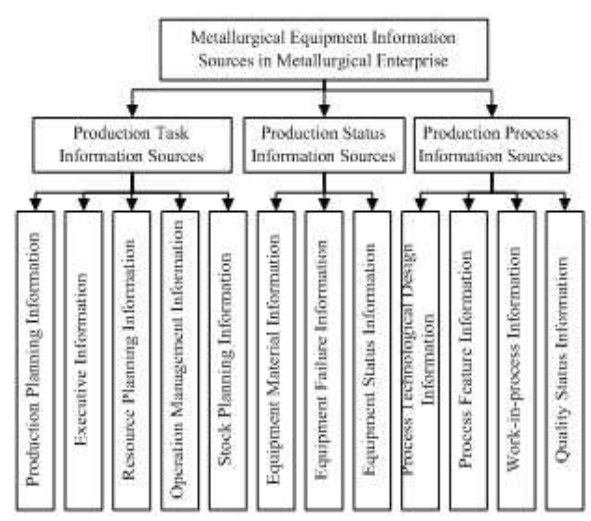

Fig. 1 Metallurgical equipment information sources within metallurgical enterprise.

\subsection{The Form of Metallurgical Equipment Information Sources in Metallurgical Enterprise}

The metallurgical equipment information sources refer to all kinds of information resources that cloud offer information for metallurgical equipment production, management and so on. According to the structure tree of the management of the metallurgy automated production line, the metallurgical equipment information sources within metallurgical enterprise can be divided into three 
categories that are production task information sources, production status information sources and production process information sources, as shown in Figure1. The metallurgical equipment information sources within metallurgical enterprise have the characteristics of distributed, heterogeneous. Therefore, to achieve the integration of ubiquitous information in iron \& steel enterprise and realize the ambient intelligence based on the integrated ubiquitous information, the unified, identical and standard management need to be executed in the management of the distributed heterogeneous metallurgical equipment information sources within metallurgical enterprise.

\subsection{Spatial Distribution of Metallurgical Equipment Production Process Information}

Metallurgical technology is complex process. The material flow, energy flow and information flow always exist in the production operation process of the metallurgical equipment. The information flow distributing in the production operation process of the metallurgical equipment do not have regularity. In order to further ascertain the spatial distribution of metallurgical equipment production process information, the process spatial information set $\mathrm{F}$ of the metallurgical process can be expressed by equation (1).

$$
F=\left|F_{i j}\right|=\left|\begin{array}{cccc}
f 11 & f 12 & \cdots & f 1 n \\
f 21 & f 22 & \cdots & f 2 n \\
\cdots & \cdots & f_{i j} & \vdots \\
f m 1 & f m 2 & \cdots & f m n
\end{array}\right|
$$

where $F$ is the process information set of the metallurgical process. Suppose $f_{i j}$ is all kinds of information space of the metallurgical process system, thus $F=\left\{f_{i j}\right\}$. Where $f_{i j}$ refers to the ith kind spatial information of process $j, i$ is a space information in the metallurgical process, $i=1, \cdots, m, j$ is a process unit in the metallurgical process, $j=1, \cdots, n$. In this way, the feature set of each process in the metallurgical process $\left\{f_{i j}\right\}$ in mathematical form can have the same dimension, if a process does not have one or more spatial information you can use[20]. In this metallurgy process system, the distributed relation of the information space between relevant process and the basic information parameter (such as production process information, production task information, equipment status information, etc) throughout the metallurgy process can be expressed by equation (2).

$$
f_{i j}=f\left(W\left(\sum \omega_{k}\right), T\left(\sum T_{k}\right), G\left(\sum G_{k}\right)\right)
$$

where $W$ is the production process information, $\omega_{k}$ is relevant factors affecting the production process information in the system, $T$ refers to the production task information, $T_{k}$ is the relevant factors of production task information in the system, $G$ is the equipment status information, $G_{k}$ is relevant factors that affect the equipment status information.

\subsection{The Information Spatial Behavior of the Metallurgical Equipment Control Process}

The information spatial behavior of the metallurgical equipment control process refers to the spatial information movement process in the space and time of the steel manufacturing process and equipment systems. The metallurgical spatial information flow of the control process is an information collection of all the information transferring from one branch (information source) to another branch (the information recipient). According to the information flow of metallurgical equipment control process (shown in Figure 2) can be known that the executive information input set (I) of executive information set $(\mathrm{E})$ of each working procedure is changed into the executive information output set $(\mathrm{O})$ by the function of various processes $\left(X_{i}\right)$. The transition from the input set to the output set is controlled by the executive information set $(E)$, the relationship information set (R) and control strategy set (C). In this process, all levels of the information subsystem (including decision-making information system, scheduling information subsystem, control information subsystem and executive information subsystem) are formed. Further more, the subsystems integrate into the decision-making center and the information flow system of the equipment control process is built[20].

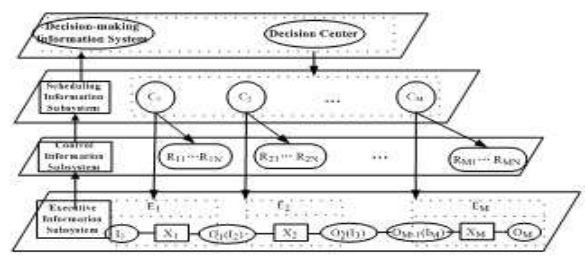

Fig. 2 The information flow of metallurgical equipment control process.

The Figure 2 shows that the bottom information flow of the metallurgical equipment control process information flow is the collection, processing, output and feedback of the information. The information terminal nodes, located in every corner of the metallurgical process, refer to the terminal signal acquisition and control equipment, the computer of the information integration and database access, the manager of processing and use the information and so on. The control system is used to control the acquisition, transmission and 
processing of the process information and endpoint control information. The control system is the data center of production organization. Equipment control system is a hub to connect the equipment scheduling system and the equipment decision-making system. The equipment scheduling system is used to split production task to bottom equipment based on equipment capacity information and equipment resources information. The production status information of the bottom equipment is given feedback to the equipment control system by the equipment scheduling system. This information will be processed or passed to the decision-making system to request processing by the equipment control system. Decision-making management system is used to make the intelligent decision according to the metallurgical equipment information integrated network and artificial intelligence processing system.

\subsection{The Metallurgical Equipment Information Spatial Scale Effect}

Spatial scale effect widely exists in the equipment information space. At different scales, the representation of the spatial patterns is different. Spatial scale determine the detailed degree of spatial dimensions and spatial aware. In order to define the scale effect of spatial information flow, the scale effect can be seen as "information flow" in the metallurgical equipment information. "Flow" can be seen as the flow of certain resource and event between a numbers of nodes connected by a network, and it is a collection of nodes, connections and resources.

Take iron and steel metallurgy for example, its process characteristics are a series of operations and processes integrated manufacturing. Information is the link of dynamically regulating and controlling various functional factors in the manufacturing process. Information also is an important foundation of the integrated optimization of the manufacturing process. The performance of the scale effect of iron and steel metallurgy equipment information flow is serialization of the production information flow in the process shaft. The ordering and continuity of the process parameters guarantee the process in different levels orderly operate and ensure to realize the continuity of the multi-factor information flow in larger scales. Since all the changes of the information exist in the process, the "process networks" structure at different levels and the function optimization of the structure in the metallurgical processes make the information flow coordinately couple to the process axis according to the specific operational procedures [21]. Only the production process information, production task information and equipment status information realizing coordination in the process spindle, can the equipment information spatial scale be controlled and can the equipment level be enhanced effectively.

\subsection{Spatial Dependence of Metallurgical Equipment Information Space}

Equipment information is often based on certain size spatial units. The equipment overall efficiency requests to conglomerate the spatial units to get the results of a larger scale unit. A certain scale-dependent exists in the multi-dimensional and multi-source spatial information.

The spatial dependence of the metallurgical equipment information space refers to the functional relationship between a spatial information unit and other units. The generation of spatial dependence means that the mutual influence of metallurgical equipment process information space is existence. In a spatial field, the observed value in a unit of the metallurgical equipment space information variable partly depends on the observed values in other units. The observed value of each variable can be expressed as $y_{i}=f\left(y_{1}, y_{2}, \cdots, y_{n}\right)$. The observed value of each variable $y_{i}$ associates with other observed values in the metallurgical equipment spatial information system by the function $\mathrm{f}$. The dependence degree can be expressed as $X \rightarrow Y(c \%, s \%, i \%)$. Where $Y$ is the spatial predicate (spatial elements or their attributes), $X$ is the collection of the predicate, $c \%, s \%, i \%$ is the credibility, support and correlation of the dependency rule. The spatial dependence of metallurgical equipment information space includes multi-scale spatial dependence, multi-layer spatial dependence and multi-dimensional spatial dependence [22].

\section{The Spatial Subdivision of Metallurgical Equipment Information Space based on CA Theory}

As an important tool for the study of complexity science, cellular automata (CA) has its own superiority, compatibility, discrete, parallel, partial, evolution and high-dimensional. The CA can well simulate the complex phenomena (such as mutations, self-organization and chaos, etc) of an open dissipative system [5].

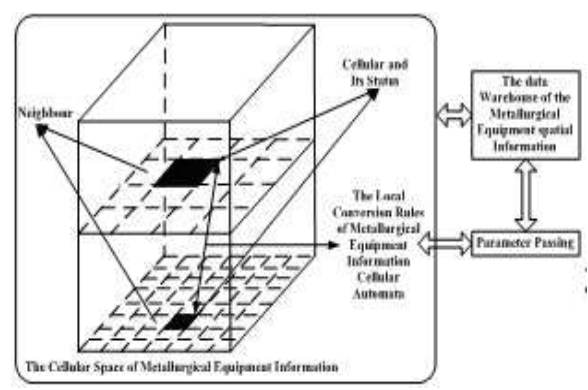

Fig. 3 The integrated framework of metallurgical equipment information space based on CA. 


\subsection{Integration Framework of Metallurgical Equipment Information Space based on The Cellular Automata}

The cellular automata are composed of 5 basic parts that is the cellular and its status, cellular space, neighbors, transformation rules and time. The parameter passing and the bilateral control of cellular information local conversion are carried out by the data warehouse. The integration framework of the cellular automata and metallurgical equipment information space is shown in Figure 3 [8].

The cellular is the most basic component of the cellular automaton, its form is $\{0,1\}$ binary or $\left\{s_{1}, s_{2}, \cdots, s_{i}, \cdots, s_{n}\right\}$ integer discrete set. The cellular space is a collection of the space networks where the cellular distributing in. The expression of cellular automata is $A=(L d, S, N, f)$. Where, $A$ represents a cellular automata system, $L$ is cellular space, the positive integer $d$ represents the cellular space dimension in cellular automata, $S$ is a limited and discrete state collection of the cell, $N=\left(s_{1}, s_{2}, \cdots, s_{i}, \cdots, s_{n}\right)\left(s_{i} \in Z, Z\right.$ is a integer set, $i \in\{1,2, \cdots, n\}$ is a composition of cells in all neighborhood (including the center cell), that is a space vector containing $\mathrm{n}$ different cellular states, and $\mathrm{n}$ is the number of neighbors of the cellular, $\mathrm{f}$ is a local transition function used to mapped $S_{n}$ to $S$. All cellular are in the d-dimensional space and their locations can be expressed by an integer matrix $Z_{i}$.

\subsection{Ubiquitous Information Space of Metallurgical Equipment Business Process Control and Intelligent Control based on Cellular Automaton}

Information flow network refers to the steel manufacturing process information system in the iron and steel metallurgy technology systems, it is necessary to divide high-dimensional information space for the information real-time control, highly integrated and optimizing of decision-making. Information space refers to the description of the specific regional location and property characteristics. Cellular spatial data is the carriers of spatial information, iron and steel metallurgy equipment cellular information space refers to form, structure, process and functional relationships of material, energy and information, and refers to its distribution pattern and its time continuation. A group element of space spread, geographical features, structure orderly and functional complementation form an interaction cellular space information collection, which is called the equipment information cellular space. It is integrity, level, differences and variability [23].

Multidimensional ubiquitous information space of intelligent control is divided based on resource space model and cellular automat theory, which is shown as Figure 4. In the figure, the information space is an overall integration of complex interrelated subsystems, it is based on ubiquitous intelligence technology and ubiquitous networks, take ubiquitous perception as core, and ubiquitous service is the target. From top to down, there are intelligent terminal-aware layer (basic automation \& process control level), network access layer and the ubiquitous intelligent manufacturing application layer (Enterprise control level). The space is also divided into the following several dimensions: X- Horizontal (task list - Quality Supervision) steel plate Life-cycle tracking information dimension, Y-(molten iron - steel plate) integrated control information dimension of plate production process, Z- Longitudinal (information perception terminal - information center server) intelligent manufacturing control information dimension of steel plate production equipment.

X- Horizontal (task list - quality supervision) steel plate life-cycle tracking information dimension. Face to metallurgical processes internal production task list, the entire enterprise information flow take metallurgical processes as the main line, each sub-module such as design, planning, production and Systems of the total system operate collaboratively and parallelly, and control the cost, quality, and progress of business orders, globally.

Y- (molten iron - steel plate) integrated control information dimension of plate production process. The steel production process is series operations, various processes, integrated manufacturing, complexity process, and there are strong dependencies and complex coupling between equipment information and time, technology, equipment, materials and so on. The horizontal metallurgy process equipment relationship set is controlled through input and output of process information.

Z-Longitudinal (information perception terminal information center server) intelligent manufacturing control information dimension of steel plate production equipment. It mainly divide control information dimension into five levels such as decision-making level, control level, scheduling levels, operating levels and physical level. Sub-information system of each level is formed and integrated into the decision-making center, and the corresponding steel plate production information transmission is formed. Interface complexity behavior and the coupled relation of each process subsystem need to be studied from the angle of plate defects intelligent control. Information terminals are distributed embedded intelligence unit, data center is the top database, and data control closed-loop system is formed through information bidirectional transmission. The intelligent manufacturing control information dimension of longitudinal steel plate production equipment is composed by intelligent terminal, information integration, sharing networks and intelligent information management and control services systems. It mainly resolve information collection, processing, storing, sharing, application, etc. among ERP, 


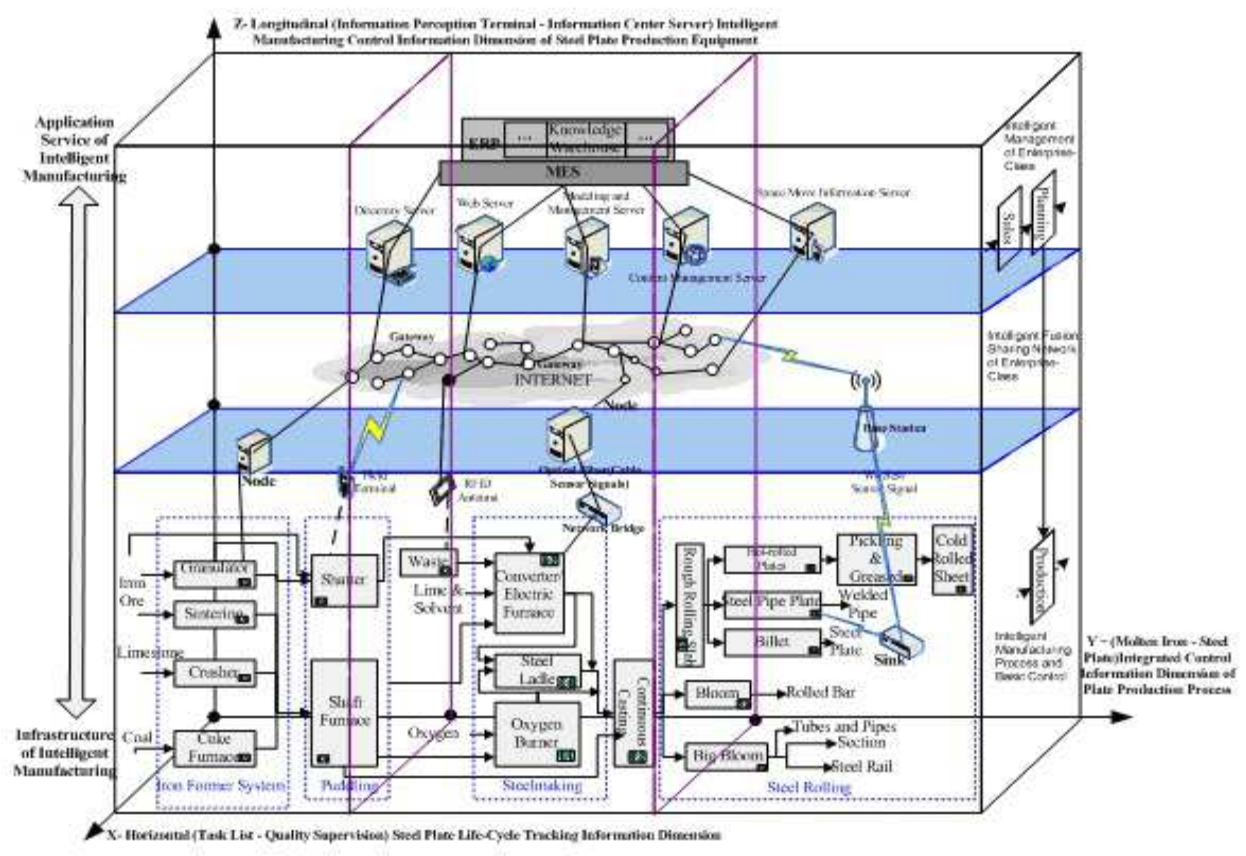

Fig. 4 Multidimensional ubiquitous information space of metallurgical equipment business intelligent control.

MES, process control systems and basic automatic control system, and improve decision-making level and equipment level.

Because the metallurgical process has strong flow characteristics, and the metallurgical process information has certain cellular characteristics. These cellular characteristics mainly reflect the time-lag effect of the cellular system. The state of a cellular at time t directly dependents on the state of the cellular and its neighbor cellular at time $\mathrm{t}-1$. At the same time, it also directly impact on its state at time $t+1$. The neighbors of the metallurgical equipment information space cellular are complex cellular neighbors in the special environment. If the metallurgical equipment information space is divided into d dimensions, the neighbors of a cellular are adjacent cellular, the number of the neighbors is $(2 d+1)^{d}-1$. The status transition function, determining the cellular status at the next time according to the current cellular state and their neighbors' status, is expressed as formula 3.

$$
f: S_{i}^{t+1}=f\left(s_{i}^{t}, S_{N}^{t}\right)
$$

where, $S_{N}^{t}$ is the neighbors' state combination at t point, $f$ is the local map or local rules of cellular automata.

\section{Multi-Point Routing Communication Paths Intelligent Planning of Metallurgical Equipment Information Space based on Ant Colony Algorithm}

The nature of multi-node routing communication in information space is to find the minimum number of connections of linking a group of nodes based on certain consideration [24]. Take metallurgical equipment information communication based on multi-node routing in equipment information space as example, communication paths intelligent planning method of iron and steel metallurgy ubiquitous intelligent systems based on equipment information space is analyzed.

\subsection{The Ant Colony Algorithm of Multi-Point Routing Communication Paths Intelligent Planning of Metallurgical Equipment Information Space}

Case problem: the barriers nodes are information transition node that are fully loaded or fault, ) the distribution of three-dimensional information space node routing network $N(V, E)$ is shown as Figure 5. To find an optimal communication path from origin point $S \in V$ to the destination point $M \in\{V-\{s\}\}$ (the length from $S$ to $M$ is $L$ ) and to ensure the communication service quality. The information communication service quality 


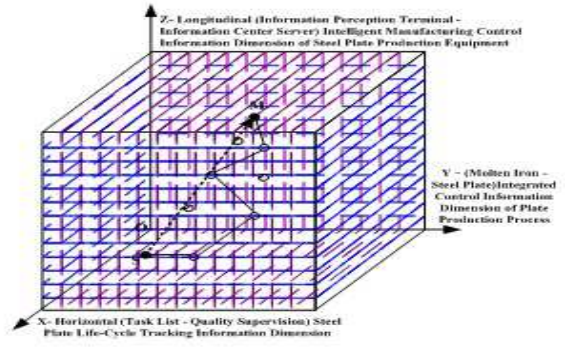

Fig. 5 The node map of the three-dimensional information space.

indicators include information delay, delay jitter, bandwidth of communication lines, packet loss rate and communication cost and so on. These factors constitute constraint condition of multi-node routing information communication in three-dimensional information space.

$V$ is a collection of all network nodes such as switches, routers and hosts, etc. In three-dimensional information space node routing network $N(V, E), E$ is the set of all edges in the graph, each edge express direct access communication path between the two adjacent nodes. Assume that the network is symmetric, and there is only an edge between the two adjacent nodes. For any information transmission link $e \in E$, there are four attributes such as information delay function (e), delay jitter function (e), communication bandwidth function (e) and communication cost function (e). For any network node $v \in V$, there are four attributes such as information delay function (v), delay jitter function (v), communication bandwidth function (v) and communication cost function (v). The information communication routing request $T(S, M)$ from the origin point $S$ to the target point $M$ exist the following relationships:

$$
\begin{aligned}
& \operatorname{delay}(T(S, M))=\sum_{e \in T\{S, M\}} \operatorname{delay}(e)+\sum_{v \in T\{S, M\}} \operatorname{delay}(v), \\
& \text { delay_jitter }(T(S, M))=\sum_{e \in T\{S, M\}} \text { delay_jitter }(e)+ \\
& \sum_{v \in T\{S, M\}} \text { delay_jitter }(v), \\
& \operatorname{cost}(T(S, M))=\sum_{e \in T\{S, M\}} \operatorname{cost}(e)+\sum_{v \in T\{S, M\}} \operatorname{cost}(v), \\
& \text { bandwidth }(T(S, M))=\min \{\text { bandwidth }(e), e \in T(S, M)\}, \\
& \text { packet_loss }(T(S, M))=1-\sum_{v \in T\{S, M\}}(1-\text { packet_loss }(v)) .
\end{aligned}
$$

In this paper, multi-point routing communication paths intelligent planning of metallurgical equipment information space based on ant colony algorithm is to find a communication path $T(S, M)$ from origin point $S$ to destination point $M$. It must meet the following two requirements:

1.delay $(T(S, M))$ is least under four constraint conditions such as delay_jitter $(T(S, M)) \leq D J$, $\operatorname{bandwidth}(T(S, M)) \geq B$, packet_loss $(T(S, M)) \leq P L$ and $\operatorname{cost}(T(S, M)) \leq C$.

2.packet_loss $(T(S, M))$ is minimum under four constraint conditions such as $\operatorname{delay}(T(S, M)) \leq D$, delay_jitter $(T(S, M)) \leq D J$, $\operatorname{bandwidth}(T(S, M)) \geq B$, and $\operatorname{cost}(T(S, M)) \leq C$.

According to the theory of ACA ant colony algorithm, to solve problem $(\operatorname{delay}(T(S, M))$ is least under four constraint conditions such as delay_jitter $(T(S, M)) \leq D J$, bandwidth $(T(S, M)) \geq B$, packet_loss $(T(S, M)) \leq P L$ and $\operatorname{cost}(T(S, M)) \leq C)$ in multi-nodes routing under the equipment information space, the ant colony algorithm process of the communication path intelligent planning is as following.

(1)Initialization of calculate information space

(I)Cartesian Coordinates system O'-X'Y'Z ', is established as Figure 6 according to the information space network $\mathrm{N}(\mathrm{V}, \mathrm{E})$ in the Figure 5 , where $S$ is origin of $\mathrm{O}^{\prime}-\mathrm{X}^{\prime} \mathrm{Y}^{\prime} \mathrm{Z}^{\prime}$ coordinates, SM direction is positive direction of $Z^{\prime}$ axis, $X^{\prime}$ axis and $Y^{\prime}$ 'axes can be the appropriately chosen. The transformation relation between Coordinate system $\mathrm{O}^{\prime}-\mathrm{X}^{\prime} \mathrm{Y}^{\prime} \mathrm{Z}$ ' and $\mathrm{O}-\mathrm{XYZ}$ is shown as ( 5)[16].

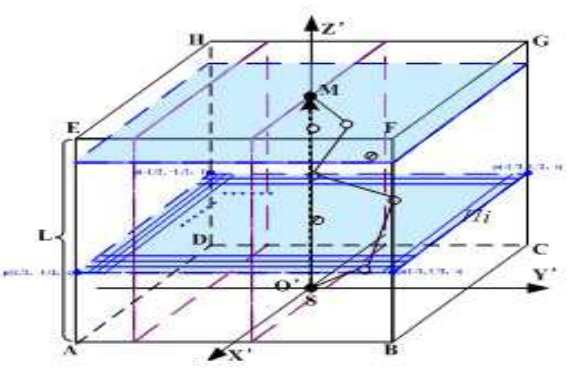

Fig. 6 The matching information space after transformating as the starting point to $S$.

$$
\left(\begin{array}{l}
x \\
y \\
z
\end{array}\right)=\left(\begin{array}{lll}
\cos \alpha_{x} & \cos \alpha_{y} & \cos \alpha_{z} \\
\cos \beta_{x} & \cos \beta_{y} & \cos \beta_{z} \\
\cos \gamma_{x} & \cos \gamma_{y} & \cos \gamma_{z}
\end{array}\right)\left(\begin{array}{c}
x^{\prime} \\
y^{\prime} \\
z^{\prime}
\end{array}\right)
$$

Where, $\alpha_{x}, \beta_{x}, \gamma_{x}$ is the intersection angle between $\mathrm{X}$-axis and $\mathrm{X}$ ', $\mathrm{Y}^{\prime} . \mathrm{Z}$ ' axis respectively, $\alpha_{y}, \beta_{y}, \gamma_{y}$ is intersection angle between $\mathrm{Y}$-axis and $\mathrm{X}$ ', $\mathrm{Y}$, ' $\mathrm{Z}$ ' axis respectively, $\alpha_{z}, \beta_{z}, \gamma_{z}$ is intersection angle between $\mathrm{Z}$-axis and $\mathrm{X}^{\prime}, \mathrm{Y}^{\prime}$. Z' axis respectively. Because the length of SM is $L$, the coordinates of $\mathrm{M}$ under coordinate system $\mathrm{O}^{\prime}-\mathrm{X}^{\prime} \mathrm{Y}^{\prime} \mathrm{Z}^{\prime}$ is $(0,0, L)$. 
(II)Make cube ABCD-EFGH in the coordinate system O'- $X^{\prime} Y^{\prime} Z^{\prime}$, the information space network $\mathrm{N}^{\prime}\left(\mathrm{V}^{\prime}, \mathrm{E}^{\prime}\right)$ is constituted as shown in Figure 6, the cube' ABCD surface is in $X^{\prime} Y^{\prime}$ plane, and it is square plane and its side length is $L$. where $\mathrm{AB} \mathrm{Y}^{\prime} \mathrm{BCX}$ ' and the origin $\mathrm{O}^{\prime}$ is in the center of the $\mathrm{ABCD}$ square plane, the high $\mathrm{AE}$ of cube is $L$, coordinates of $\mathrm{M}$ is $(0,0, L)$. O'M' is divided into $(n+1)$ equal portions, $n$ planes $\prod_{i}(i=1,2, \cdots, n)$ perpendicular to the $Z^{\prime}$ axis are made over each equal point. The square plane $i$ is the cross-section of Plane $i$ intersect cube ABCD-EFGH as shown in Figure 6, square plane $\prod_{i}(i=1,2, \cdots, n)$ is divided into $\mathrm{mm}$ small square. For the square vertex $p(u, \omega, i) \in V^{\prime}(u, \omega=0,1, \cdots, m)$ of square plane $\prod_{i}, \quad$ its actual coordinates is $\left(-\frac{L}{2}+\frac{u * L}{m},-\frac{L}{2}+\frac{\omega * L}{m}, \frac{i * L}{n}\right)$ in the coordinate system of O'-X'Y'Z'.

(II)Suppose that there are $W=\left(w_{1}, w_{2}, \cdots, w_{n}\right)$ to be transfer information of intelligent terminal in network space, N'(V', E'), which is transmitted from the origin point $S(0,0,0)$. Set $\operatorname{delay}(p)$,delay_jitter $(p)$, packet $\operatorname{loss}(p), \operatorname{cost}(p)$ of each routing node $p(u, \omega, i) \in V^{\prime}$ in network space N-'(V', E'), set $\operatorname{delay}\left(e^{\prime}\right), \operatorname{delay}$ jitter $\left(e^{\prime}\right)$

[25],

bandwidth $\left(e^{\prime}\right), \operatorname{cost}\left(e^{\prime}\right)$ of each edge $e^{\prime} \in E^{\prime}$, then set constraints $D J, B, P L$ and $C$, and the Maximum iterations is DDmax.

(IV)Calculate all points' allowed list allowed $(u, \omega, i)(u, \omega=0,1, \cdots, m)$ on the plane $\prod_{i}(i=1,2, \cdots, n-1)$. Suppose $p(u, \omega, i)$ is one point of plane $\prod_{i}(i=1,2, \cdots, n-1)$, to any point $p(k, q, i+1)(k, q=0, l, \cdots, m)$ on the plane $\prod_{i+1}$, if node $p(k, q, i+1)$ is trouble-free and information on line $p(u, \omega, i), p(k, q, i+1)$ can be transferred successfully, then $p(k, q, i+1)$ point is added to allowed list allowed $(u, \omega, i)$. According to this method, we can calculate all the allowed reach points of $p(u, \omega, i)$, and store them into the allowed list allowed $(u, \omega, i)$. Remove all the nodes outside the allowed list and the links do not meet the bandwidth constraints.

(V)Initialize the pheromone list $\tau_{u \omega}^{i}$ of all points $p(u, \omega, i)(u, \omega=0,1, \cdots, m)$ on plane $\prod_{i}(i=1,2, \cdots, n-1)$. Pheromone list is an array, where each data is used to represent the connection strength of pheromone between point $p(u, \omega, i)$ and point $p(k q i+1)$. Suppose Initiate pheromone $\tau_{u \omega}^{i}(0)=A, \Delta \tau_{u \omega}^{i}(0)=0$, where $A$ is a constant.

(2)In every step of the information dissemination path building, point $p(u, \omega, i)$ in coordinate system O'-X'Y'Z' determine the next node of information dissemination based on the heuristic information value and pheromone, it is shown as (4). Where $j$ is a random variable obey probability distribution according to the formula (6), $r$ is a constant of section $[0,1], r_{0}$ is uniform distribution random number of section $[0,1]$.

$$
p_{i+1}=\left\{\begin{array}{c}
\arg \max _{(k, q, i+1) \in \text { allowed }(u, \omega, i)}\left[\tau_{i+1} \bullet \eta_{i, i+1}\right], r<r_{0} \\
J \quad, r>r_{0}
\end{array}\right.
$$

To pending transfer information of any point $p(u, \omega, i)$ on plane $\prod_{i}(i=1,2, \cdots, n)$, the select probability of point $p(k, q, i+1)$ on plane $\prod_{i+1}$ is shown as formula (6).

$$
p_{i, i+1}=\left\{\begin{array}{cc}
\frac{\tau_{i+1} \bullet \eta_{i, i+1}^{\beta}}{\sum \tau_{i+1} \bullet \eta_{i, i+1}^{\beta}}, p(k, q, i+1) \in \text { allowed } \\
0 \quad p(k, q, i+1) \notin \text { allowed }
\end{array}\right.
$$

Where, $\tau_{i+1}$ is pheromone amount stored by point $p(k, q, i+1)$ on plane $\prod_{i+1}, \eta_{I, i+1}=1 / d\left(p_{i}, p_{i+1}\right)$ is heuristic function, $\beta=\left\{\begin{array}{c}(4 t-2 r) / t, 0 \leq r \leq t \\ 2, t \leq q\end{array}\right.$ is heuristic factor and $t$ is the critical time, which reflect the respect degree of heuristic information in the process of information transfer path selection [14].

(3)Whenever Information is delivered to a node, $\tau_{u \omega i}=$ $(1-\mu) \tau_{u \omega i}+\mu \tau_{0}$ is called immediately to update local information in real time. If the information can not find the next node after a node is reached, it is considered that the information is void.

(4)Determine whether the trans-information meet at certain node, and if so, meet strategy operating is do according to $L=L\left(w_{1}\right)+L\left(w_{2}\right)\left(w_{1}, w_{2}\right.$ meet) to produce a new path and the path is placed into the path table. Then it is updated in accordance with the formula $\tau=(1-\mu) \bullet \tau+\mu \bullet \Delta \tau$, where $\Delta \tau=1 / L_{\text {new }}, \mu$ is one parameter among $0-1, \tau_{0}$ is pheromone initial value of each feasible point. Otherwise, go to (2).

(5)Global pheromone is updated in accordance with the formula $\tau_{u \omega i}=(1-\rho) \bullet \tau_{u \omega i}+\rho \bullet \Delta \tau_{u \omega i}$, where $\rho(0<\rho<1)$ is the pheromone evaporation coefficient, $1-\rho$ is the degree of pheromone attenuation with time, $\Delta \tau_{u \omega i}=\sum_{i=1}^{W} \Delta \tau_{u \omega i}^{w}$ is information increase of each path after every iteration, where $\Delta \tau_{u \omega i}^{w}=1 / L_{\mathrm{w}}$, and the $\mathrm{w}$ information goes through node $p(u, \omega, i)$ in current transfer process, otherwise $\Delta \tau_{u \omega i}^{w}=0$. Determine whether the algorithm satisfies the stop condition, if so, optimal results are output; otherwise go to (2). Until all pending transfer information completes the steps (2), it is to say that route from $\mathrm{S}$ to $\mathrm{M}$ is found or void.

\subsection{Simulation Analysis}

In this paper, simulation software Matlab7.0 is used to simulation analysis the optimal communication path of 
equipment information multi-point routing delay $(T(S, M))$. Attribute parameter of each routing node is expressed by delaydelay jitterpacket loss and cost. Attribute parameter of each link is expressed by delaydelay jitterbandwidth and cost. The simulation experiment parameters are set as $W=30, D D \max =$ $100, \rho=0.8, L=5, D J=15, B=60, P L=0.01, C=10$.

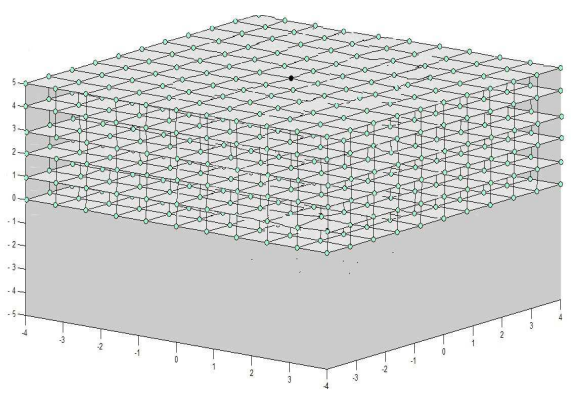

Fig. 7 Multi-point routing communication path of the threedimensional information space

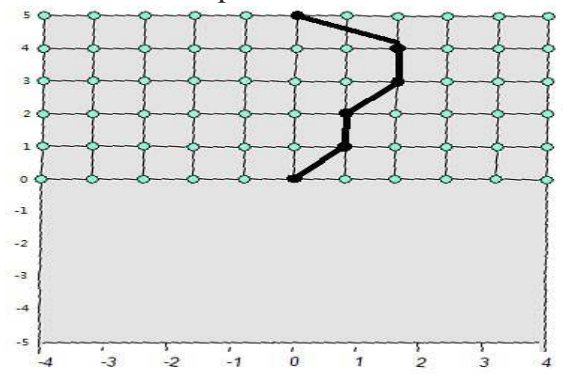

Fig. 8 The projection of the communication path in X'Z' .

To routing requests from source point $S(0,0,0)$ to $M(0,0,5)$ based on space network $N^{\prime}\left(V^{\prime}, E^{\prime}\right)$ shown as Figure 7 , its optimal path is " $S(0,0,0) \rightarrow p(1,2,1) \rightarrow$ $p(1,2,2) \rightarrow p(2,4,3) \rightarrow p(2,2,4) \rightarrow M(0,0,5) "$ shown in Figure 8. To the optimal path, the Delay jitter is 12, cost is 20, information loss rate is 0.00289, and information delay is 10 .

\section{Conclusions}

Metallurgical equipment information space is complex and diverse. The multi-level, multi-dimensional and multi-source characteristics of the metallurgical equipment information space are often ignored. The existing spatial analysis technique is restricted to two dimensions. From the perspective of the composite analysis of the spatial information, the metallurgical equipment information spatial characteristics (such as the space distribution, spatial morphology, spatial behavior, spatial scale effects and space dependence, etc) are compositely analyzed based on the resource space model and cellular automata theory. In the support of the metallurgical equipment information source and the drive of information flow, the integrated framework of metallurgical equipment information space is built based on the cellular automata. The ubiquitous information spaces of metallurgical equipment business process management and intelligent control are divided. In order to improve the quality of the information space communications services, information communication path intelligent planning method of metallurgical equipment based on ACO algorithm is explored. The simulation results show that the delay jitter, the information loss rate and information delay of optimal path optimal verify. The practicality and effectiveness of the divided information spaces and designed algorithms are verified by simulation, which provides theoretical support to enhance the level of metallurgical equipment.

\section{Acknowledgement}

This work is partially supported by the general Program of the National Natural Science Foundation of China (Grant No. 51174151), National" Twelfth Five-Year" scientific and technological support plan subject(no. 2011BAB05B02). Thanks for the help.

\section{References}

[1] P Zhang, Y Ji, Y.N Li, J. ZTE Communications 3, (2007).

[2] H.X Liu, J. Library and Information Service 22, 47-50 (2010).

[3] L Zhen, Doctoral dissertation of Shanghai Jiaotong University. (2008).

[4] Z.W, Yuan, S.B Jia, J. Computer Engineering 7, 61-65 (2010).

[5] L.X Yang, The doctoral dissertation of the PLA Information Engineering University, 2004.

[6] D.S Sun, J. Wuyi University (Natural Science).4, 22-28 (2011).

[7] J Shi, Z Chen, J. Journal of Sensors and Actuators 12,17341738 (2011).

[8] S.F Wei, J.L Li, Z.F Shao, J. Computer Engineering and Applications 9, 4-7 (2007).

[9] S.T Liu, S.Q Yang, J. Infrared and Millimeter Waves 1, 42 46 (2008).

[10] M Wang, J Zhao, J. Materials Review 11, 133-135 (2011).

[11] F.C Xue, PhD thesis of China University of Mining and Technology, 2008.

[12] G. Tigan, J. Appl. Math. Inf. Sci. 4, 383-394 (2010).

[13] J.H Gao, R. Shan, J. Appl. Math. Inf. Sci. 6, 35-39 (2012).

[14] H Hu, X.S Cai, J. Computer Systems \& Applications 11, 95-98 (2011).

[15] K.H Lee, K. Oh, J. Appl. Math. Inf. Sci. 6, 311S-321S (2012).

[16] X.B Hu, X.Y Huang, J. Chongqing University 8, 132-135 (2004). 
[17] X.Q Xie, C.C Song, Z.Q Zhang, J. Computers 11, 20932103 (2010).

[18] F Yu, W.H Liao, Y.N Xie, Y. Guo, J. Computer Aided Design and Computer Graphics 7, 952-956 (2008).

[19] M Frehner, M Brandli , JEnvironmental Modeling \& Software 11, 1544-1554 (2006).

[20] R.Y Yin, Metallurgical process engineering, M. Metallurgical Industry Press, 2009.

[21] N Zhang, J. Appl. Math. Inf. Sci. 6, 129-133 (2012).

[22] M.H Pan, J. Doctoral thesis of Chinese Academy of Sciences. 2006.

[23] H.B Max, The learning framework of information space - organizations, institutions and culture, M. Shanghai: Shanghai Translation Publishing House, 2000.

[24] M. Dorigo, E Bonabeau, G. Theraulaz, Ant algorithms and stigmergy, J. Future Generation Computer Systems, 2000.

[25] J.O Alzabut, T Abdeljawad, J. Appl. Math. Inf. Sci. 5, 74-84 (2011).

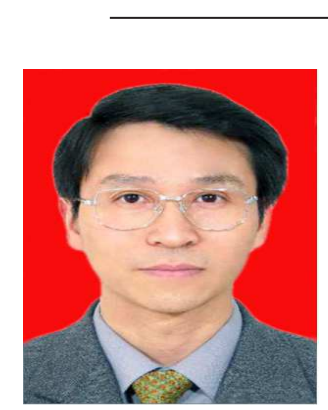

Jianyi Kongis a professor, doctoral supervisor and the president of Wuhan University of Science and Technology. He received the MS degree from Xi'an Jiaotong University in 1984, and the $\mathrm{PhD}$ degree from Universit?t der Bundeswehr Hamburg in 1995. He has served as a visiting professor of Universität der Bundeswehr Hamburg. His research interests are in the areas of intelligent machine and controlled mechanism, mechanical and electrical system dynamic design and fault diagnosis, mechanical $\mathrm{CAD} / \mathrm{CAE}$, and Intelligent design and control.
Junwei

Liu

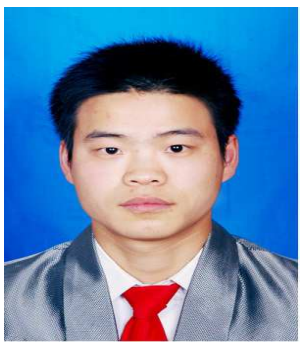

received the Master degree in Machinery Manufacturing and Automation from Wuhan University of Science and Theology in 2009. He is currently a doctoral student of Mechanical Engineering in Wuhan University of Science and Technology. His research interests are in the areas of manufacturing informatization and intelligent manufacturing.

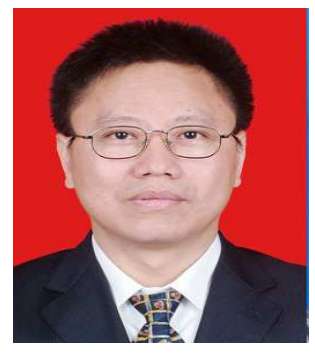

Yongbing $\mathbf{L i}$ received the Master degree in systems engineering from Xi'an Jiaotong University in 1989 , has been engaged information system construction work in the Wuhan Iron and steel enterprise. His main research area is the overall production and marketing information systems based on centralized management- multistory structure.

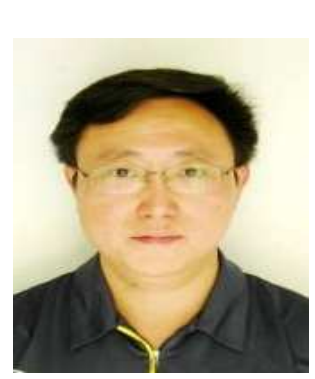

Wang is a professor at Wuhan University of Science and Technology. His research interests are in $\mathrm{CAD} / \mathrm{CAM} / \mathrm{CAE}$ and industrial automation testing. 


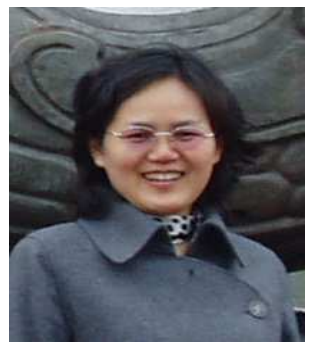

Min Zhou is a professor in the College of Machinery and Automation and the dean of Industrial Engineering department at Wuhan University of Science and Technology. She received the $\mathrm{PhD}$ degree in Management Science and Engineering from the Wuhan University of Technology in 2006. Her

research interests are in manufacturing informatization, knowledge management and engineering and equipment management and engineering. 